\title{
Editoriale
}

\section{Lock per CVC: realtà e nuove prospettive}

\author{
G. Forneris \\ S.C. Nefrologia e Dialisi, Ospedale S. Giovanni Bosco, Torino
}

CVC AND LOCK: STATE OF ART AND NEW PERSPECTIVES

ABSTRACT. CVC lock represents an actual basic theme in hemodialysis vascular access field. Recent know-how about biofilm led lock copyright holder towards warranty both in preventing infections endorsing CVC performance at the same time. Still today, an ideal lock, with antithrombotic and anti-bacterial properties, is not yet available. Today, with its pros and cons, heparin represent most employed lock even though physicians are looking for alternative devices. Among them, binders employment, such as low concentration sodium citrate (3.8 - 4\%) could be effective for safety and low costs. High concentration sodium citrate (46.7\%), taurolidine and ethanol can be considered as "advanced" locks with their distinctive features and potential further employment. Anyway, infections' prevention protocols adhesion and accurate CVC management represent primary target of daily hemodialysis practice irrespective of lock's choice.

KEY WorDs. Lock, Citrate, Central Venous Catheter, Biofilm

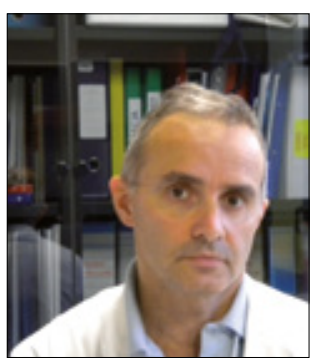

Giacomo Forneris
In questo numero di $T N \& D$ Salvatore Mandolfo, da molti anni attento conoscitore della problematica del lock per i CVC da emodialisi, offre un aggiornamento su una materia di certo interesse come testimoniano $\mathrm{i}$ numerosi articoli pubblicati su prestigiose riviste. Il lock è tuttavia un tema conosciuto spesso superficialmente dagli stessi utilizzatori, che meccanicamente ripetono l'instillazione della soluzione di stazionamento interdialitica. È quindi una scelta editoriale felice offrire una messa a punto sulla situazione attuale e sulle prospettive future. Il lavoro illustra in modo ordinato vantaggi e limiti delle soluzioni lock di cui oggi disponiamo e le differenze di uso nella profilassi e nel trattamento sul versante antitrombotico e nei confronti delle infezioni. Dal titolo emerge tuttavia come siamo ancora lontani, per usare un gioco di parole, dalla "soluzione": non disponiamo cioè di un lock ideale provvisto di proprietà antitrombotiche e allo stesso tempo profilattiche nei confronti delle infezioni, durature nel tempo e prive di effetti collaterali. Gli utilizzatori, in primis il personale infermieristico che riveste il ruolo più importante nella gestione quotidiana dei CVC, sanno che oggi la scelta del lock ricade quasi sempre in prima battuta sull'eparina o sul citrato a bassa concentrazione. Nell'articolo Mandolfo descrive in modo ordinato pregi e svantaggi di ciascuno di questi due lock, affrontando successivamente la possibilità di utilizzo dei lock "alternativi”. L'occasione mi permette di aggiungere all'esperienza dell'autore quella personale che è paradigmatica e probabilmente una delle prime in Italia nel senso di un cambiamento radicale. Da oltre dieci anni presso la SC dell'Ospedale S. Giovanni Bosco di Torino il protocollo di gestione del CVC prevede l'uso del citrato a bassa concentrazione (3.8\%), con l'esclusione totale dell'eparina da tutti i CVC, compresi i non tunnellizzati in uso specialmente in rianimazione per le CRRT. La scelta a suo tempo intrapresa in risposta ad alcuni episodi di batteriemia, prescindeva da molte delle conoscenze emerse negli anni successivi, in particolare del ruolo del biofilm nelle infezioni a partenza endoluminale e del comportamento dei chelanti e dell'eparina su questo sistema affascinante e complicatissimo. Se questa scelta abbia influito sugli ottimi risultati ottenuti nei confronti delle infezioni resta speculativo ma di certo alcuni dei possibili problemi legati all'eparina, frequentemente riscontrati in precedenza, non sono stati più osservati. L'opinione personale è perciò in pieno accordo con le conclusioni dell'articolo di 
come il lock a base di eparina confermi ormai troppi svantaggi rispetto al citrato a bassa concentrazione, suffragando dunque la nostra scelta. Quanto questa opinione o questa scelta sia condivisa e soprattutto messa in atto nei Centri dialisi italiani non è noto. $\mathrm{E}$ verosimile che l'utilizzo del citrato a bassa concentrazione sia in aumento ma che la maggior parte dei nefrologi sia di fatto ancora legato all'eparina, spesso solo per tradizione. Questo trend di cambiamento è sostenuto dai suggerimenti di alcune società scientifiche, non solo in ambito nefrologico ma anche nel campo dei CVC utilizzati nelle ICU, dove il messaggio "replace heparin with chelators" ha recentemente avuto una notevole eco. Il recupero del dato sull'utilizzo dei diversi lock in Italia rappresenta pertanto una necessità conoscitiva della realtà degli accessi vascolari e l'auspicio è che il Gruppo di Studio possa farsene carico nel prossimo futuro. Nell'attesa, il ricorso a soluzioni lock "alternativi o avanzati", in grado di offrire una maggiore protezione antitrombotica e antimicrobica ha avuto alterne fortune. Tra questi il tentativo di aumentare la concentrazione del chelante ha deluso in parte le aspettative negli studi clinici, confermando in vivo e in vitro i rischi. Resta nella convinzione personale che "primum non nocere" dovrebbe rimanere un criterio prioritario nella nostra professione in presenza di alternative, se non più efficaci, almeno prive di rischi. L'etanolo, a fronte di una potente efficacia dimostrata nel potere antimicrobico, ha un giudizio in sospeso sulla possibile induzione di malfunzionamento del CVC, le conseguenze a lungo termine sul materiale, gli effetti collaterali dello spillage dell'alcol e non ultimo, il fatto che chi intende utilizzarlo deve farlo sotto la propria responsabilità. L'ultimo commento sui lock alternativi riguarda la taurolidina, che ha dimostrato in quasi tutti gli studi, compresa la nutrizione parenterale e l'oncoematologia, elevata efficacia e assenza di eventi avversi, ma la cui esperienza è stata finora limitata dai costi del dispositivo, in un periodo economicamente non proprio favorevole. Non sfugga come anticipato l'importanza del fenomeno dello spillage, cioè la perdita precoce di una parte della soluzione appena instillata nel CVC, che passa direttamente nel torrente circolatorio. Oltre ai possibili effetti collaterali specifici di alcune soluzioni, va ben tenuto a mente che questo fenomeno interessa soprattutto la punta del catetere in modo differente in base alla tipologia costruttiva, e ciò rappresenta in ogni caso un impedimento per una completa protezione del lock anche qualora provvisto di caratteristiche ideali. In questo senso, la prospettiva di realizzazione di un lock mec- canico, costituisce una delle sfide più stimolanti nel futuro dei CVC. Infine per coloro che al momento ricorrono in diversa misura ai lock alternativi, rimane aperto il problema delle indicazioni, cioè dei pazienti che potenzialmente ne trovano vantaggio a fronte di un maggiore impegno economico. Infatti una scelta radicale di un utilizzo esteso non è forse oggi giustificabile considerando che ottimi risultati possono essere ottenuti con la sola applicazione delle misure di prevenzione generali. Le Guidelines CDC 2011 suggeriscono l'utilizzo di un lock alternativo profilattico in presenza di una storia di infezioni ricorrenti nonostante l'adozione corretta delle altre misure di prevenzione. Potrebbe essere però interessante chiarire eventuali vantaggi in prevenzione primaria in altre categorie di pazienti come quelli il cui CVC in uso rappresenta un accesso senza più alternative, o quelli affetti da stati gravi di immunodepressione ad alto rischio di infezione. Un breve commento infine sul lock terapeutico, cioè l'instillazione di una soluzione lock antimicrobica per il trattamento delle batteriemie da CVC, in associazione alla terapia antibiotica sistemica. La Lock Therapy rientra da tempo nelle linee guida ma la disponibilità di protocolli per la preparazione con diversi tipi di antibiotico non è diffusa in modo capillare. La preparazione di queste soluzioni può nascondere delle insidie come si legge nell'articolo e non deve essere improvvisata di volta in volta: sia dunque il nostro personale infermieristico di stimolo per colmare questa lacuna là dove presente. Per il lettore che non ha avuto modo di affrontare negli ultimi tempi il tema del lock, la lettura dell'articolo di Mandolfo è una buona occasione per appropriarsene rapidamente ed appassionarsi, ma mi auguro che serva anche a mettere in discussione il proprio modo di lavorare, confermandolo là dove $\mathrm{i}$ risultati danno ragione, ma avendo se necessario il coraggio di cambiare.

\section{Riassunto}

Il lock dei CVC rappresenta una tematica estremamente attuale, in particolare nel campo degli accessi vascolari per dialisi. Le recenti conoscenze sul biofilm hanno spostato l'interesse dalla proprietà del lock di garantire la pervietà del CVC a quella di prevenire le infezioni. Tuttavia non abbiamo ancora a disposizione un lock ideale dotato di proprietà antitrombotiche e protettive nei confronti delle infezioni. L'eparina rappresenta il lock tradizionalmente più utilizzato anche se nel corso del tempo ha mostrato 
alcuni aspetti sfavorevoli e stimolato la ricerca di soluzioni alternative. Tra queste l'uso dei chelanti, in particolare del citrato di sodio a bassa concentrazione (3.8-4\%) è quella che riscuote attualmente il maggiore consenso per la sicurezza di impiego e i costi ridotti. Citrato ad alta concentrazione (46.7\%), taurolidina ed etanolo rappresentano invece lock avanzati, ciascuno con peculiari caratteristiche e prospettive di futuro impiego. In ogni caso l'aderenza alle misure di prevenzione generali nei confronti delle infezioni e la corretta gestione del CVC costituiscono la condizione imprescindibile per l'attività quotidiana indipendentemente dalla scelta del lock.

Parole Chiave. Lock, Citrato, Catetere Venoso Centrale, Biofilm
Indirizzo degli Autori:

Dr. Giacomo Forneris

SC Nefrologia e Dialisi

Ospedale S. Giovanni Bosco

Piazza del Donatore di Sangue 3

10154 Torino

gforneris62@gmail.com 